\title{
Materials chemistry at Nankai University: A special issue dedicated to the 100th anniversary of Nankai University
}

\author{
Dongbing Zhao ${ }^{1}$, Jialiang $\mathrm{Xu}^{2}, \mathrm{Xian}-\mathrm{He} \mathrm{Bu}^{1,2^{*}}$ and Jun $\mathrm{Chen}^{1^{*}}$
}

It is our great honor to dedicate this special issue of Science China Materials to Nankai University (NKU) in celebration of its 100th anniversary. Established by two famous patriotic educators Xiu Yan and Boling Zhang in 1919, NKU is one of China's top universities with a long tradition in education. It has been playing essential roles as a pioneer in the course of China's modernization with the aim of "Knowing China and Serving China". Chemistry at NKU has a rich history. Since its founding, Chemistry at NKU has been leading the development of outstanding chemists and produced high-impact scientific discoveries. NKU Chemistry has continuously opened up new fields and built up new academic institutions in response to the major developmental needs of the country. In line with the requirements of national advanced material developmental strategy, NKU established the discipline of materials science in 1999 by integrating the strengths of chemistry and physics in the field of advanced materials, and founded the School of Materials Science and Engineering and the National Institute for Advanced Materials in 2015. With development over a decade, Material Chemistry at NKU blossomed and became fruitful. This special issue includes 13 review papers and 1 perspective, covering both traditional and emergent topics as a testament to the breadth of this discipline at NKU.

For energy conversion and storage materials, Zhenhua Yan et al. (https://doi.org/10.1007/s40843-019-9464-0) have discussed on the structure design and working mechanism of silicon anode for lithium-ion batteries, while Zhong'an Li, Dongbing Zhao et al. (https://doi.org/ $10.1007 /$ s40843-019-9465-4) focused on the rhodanine- based nonfullerene acceptors for organic solar cells. Meanwhile, Xiaohong Liu, Tianying Yan et al. (https:// doi.org/10.1007/s40843-019-9458-3) have reviewed recent progress in the ionic liquid electrolytes in electric double layer capacitors, with special attention to the relationship between the structures of different ionic liquid-based electrolytes and the energy storage properties in electric double layer capacitors. Li Li, Yijing Wang et al. (https:// doi.org/10.1007/s40843-019-9556-1) have reviewed the progress, prospects and challenges in the area of lightweight hydrides nanocomposites for hydrogen storage. With the enormous research on electrochemical energy storage devices, the safety issue of such devices has appealed main concern. Jialiang Xu et al. (https://doi.org/ 10.1007/S40843-019-9475-4) thus paid special attention to the safety regulation of gel electrolytes and envisaged the challenges and directions in terms of the development of gel electrolytes.

Luminescent metal-organic frameworks (LMOFs) are emerging as chemical sensors for detection of diverse targets. The relative progresses and detection mechanisms of LMOFs have been discussed by Xian- $\mathrm{He} \mathrm{Bu}, \mathrm{Na} \mathrm{Li}$, et al. (https://doi.org/10.1007/s40843-019-1169-9), directing the construction and sensing applications of LMOFs. The study on nano-scale materials has drawn immense attention to electrohydrodynamic nanowire printing technique and copper nanomaterials and their assemblies for applications on soft electronics, which are summarized by Wentao Xu et al. (https://doi.org/10.1007/s40843-0199583-5) and Jian Zhu et al. (https://doi.org/10.1007/ s40843-019-9468-5), respectively.

For catalytic materials, Tiehong Chen et al. (https://doi.

\footnotetext{
${ }^{1}$ College of Chemistry, Nankai University, Tianjin 300071, China

${ }^{2}$ School of Materials Science and Engineering, National Institute for Advanced Materials, Nankai University, Tianjin 300350, China

* Corresponding authors (emails: buxh@nankai.edu.cn (Bu XH); chenabc@nankai.edu.cn (Chen J))
} 
org/10.1007/S40843-019-9476-5) have summarized (https://doi.org/10.1007/s40843-019-9468-5) carbon encapsulated $\mathrm{Fe} / \mathrm{Co}$-based nanoparticles catalysts for oxygen reduction reaction (ORR), while Hongbo Zhang et al. (https://doi.org/10.1007/s40843-019-9454-x) have reviewed the recent advances in selective $\mathrm{C}-\mathrm{C}$ bond coupling for ethanol upgrading over balanced Lewis acidbase catalysts.

In the area of biomedical materials, Yaping $\mathrm{Du}$ and Xinyun Zhai et al. (https://doi.org/10.1007/s40843-0199471-7) shed light on the mechanisms of the antibacterial activity of cerium oxide nanoparticles. Potential applications and prospects are proposed to serve as a guidance for the design of antibacterial materials. Dan Ding, Xue Xue and colleagues (https://doi.org/10.1007/s40843-0199470-3) traced the advances in organic/polymer photothermal nanoagents towards in vivo photoacoustic imaging and photothermal therapy. Furthermore, immunotherapy aimed for cancer has also been widely explored. Zhilin Yu et al. (https://doi.org/10.1007/s40843019-9451-7) contributed their endeavor to sum up the evolution of cancer immunotherapy based on peptide therapeutics and assemblies, particularly highlighting the peptide-based nanomaterials as scaffolds for targeting delivery or co-delivery of multiple therapeutics to enhance immunogenicity. Wei Li et al. (https://doi.org/ 10.1007/s40843-019-1177-y) systematically summarized the intrinsic relationship between the crystal structure and mechanical properties of the coordination polymer dietary supplement, and compared it with small molecule drug crystals and inorganic dietary supplements to provide a reference for its tablet processing and manufacturing process.

We selected these papers mainly from the School of Materials Science and Engineering and the College of Chemistry as well as other departments of NKU in the hope of enhancing academic exchanges and cooperation both at home and abroad, contributing to the development of materials chemistry and related interdisciplinary research.

We would like to express our deep appreciation to Prof. Yadong Li, the Editor-in-chief of Science China Materials, to give us the opportunity to organize this special issue. We would also like to thank Dr. Guilin Wang (the Managing Editor), as well as all the staff at Science China Materials for their professional efforts dedicated to this special issue.

Received 24 September 2019; accepted 24 September 2019; published online 26 September 2019

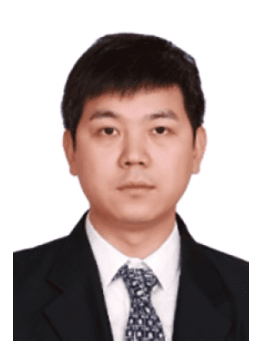

Dongbing Zhao is a professor of chemistry at the College of Chemistry of Nankai University. He obtained his PhD from the Chemistry Department at Sichuan University under the supervision of Professor Jingsong You in 2012. From 2012 to 2016, he was a postdoctoral research fellow in organic chemistry at Muenster University, in polymer chemistry at Cornell University and in material science at University of Washington. He started his independent career at Nankai University in 2016 in the College of Chemistry. His research interests include organic synthesis, $\pi$-conjugated materials, bio-images and organic solar cells.

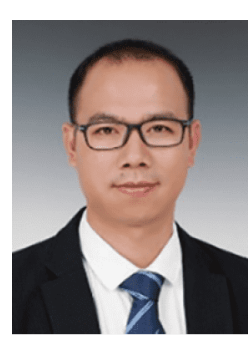

Jialiang $\mathrm{Xu}$ is a professor of materials chemistry at the School of Materials Science and Engineering of Nankai University. He obtained his $\mathrm{PhD}$ from the Institute of Chemistry, Chinese Academy of Sciences in 2010 under the supervision of Prof. Yuliang $\mathrm{Li}$, and then worked as a Marie-Curie Fellow at Radboud University, Nijmegen, hosted by Prof. Alan Rowan and Prof. Theo Rasing. In 2013, he was awarded the NWOVENI grant, with which he developed his own research line at the interface between chemistry and physics to study the coupling between light and (supra)molecular systems. In 2015, he returned to China and joined the School of Chemical Engineering and Technology at Tianjin University. He relocated to Nankai University in 2018.

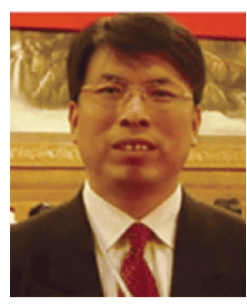

Xian-He Bu received his $\mathrm{BSc}$ and $\mathrm{PhD}$ degrees from Nankai University in 1986 and 1992, respectively, under the supervision of Prof. Yun-Ti Chen. He was promoted to a full professor in 1995. In 2002, he won the support of the $\mathrm{Na}$ tional Outstanding Youth Foundation; in 2004, he was selected as Cheung Kong Scholar Professor by the Ministry of Education. He is now the dean of the School of Materials Science and Engineering of Nankai University. He won the second prize in the National Natural Science Award in 2014 and the grand prize in Tianjin Natural Science Award in 2018. His research focuses on functional coordination chemistry, crystal engineering, and molecular magnetism.

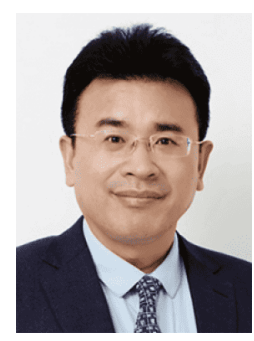

Jun Chen is a professor at Nankai University, and the Academician of the Chinese Academy of Sciences. He serves as Vice President of Nankai University. He received his Bachelor's and Master's degrees from Nankai University in 1989 and 1992, respectively, and his doctoral degree from Wollongong University, Australia, in 1999. He has been engaged in the chemical research of new energy materials and made important innovative contributions. He has published more than 400 SCI papers in scientific journals such as Nature Chemistry and has been granted 30 invention patents, some of which have been applied to real production. He won second prize in the National Natural Science Award in 2011 and the National "May First" Labor Medal in 2018. 